\title{
Management of Acute Coronary Syndrome and Venous Thromboembolism: A Managed Care Perspective
}

\author{
Randall D. Seifert, PharmD
}

\begin{abstract}
BACKGROUND: Acute coronary syndrome (ACS) and venous thromboembolism (VTE) are costly conditions, largely due to the high initial cost of treatment, patient nonadherence to prescribed antiplatelet therapy, avoidable rehospitalization in patients with ACS, and high rate of recurrence and long-term complications in patients with VTE.

OBJECTIVES: To discuss the economic impact of ACS and VTE; factors that contribute to preventable morbidity, mortality, and costs associated with these conditions; and strategies that managed care pharmacists can use to improve clinician knowledge of evidence-based treatment guidelines and patient adherence to the treatment plan, thereby improving clinical and economic outcomes.
\end{abstract}

SUMMARY: Premature discontinuation of antiplatelet therapy increases the risk of death and rehospitalization in patients with ACS. Factors associated with premature discontinuation include advanced age, lack of education, unmarried status, pre-existing cardiovascular disease, high cost of health care, failure to receive discharge instructions, and lack of referral for cardiac rehabilitation. Managed care plan benefit design should provide for the effective treatment of ACS and VTE by identifying the optimal type and duration of anticoagulant and antiplatelet therapy. In patients with VTE, the use of low-molecular-weight heparin (LMWH) in outpatients is as safe and effective and less costly than standard intravenous unfractionated heparin on an inpatient basis. Long-term LMWH treatment for acute deep vein thrombosis is safe and effective, and it is preferred over warfarin for patients with cancer. Managed care pharmacists can improve the quality of care for patients with ACS and VTE by using a variety of strategies to improve clinician knowledge of evidence-based treatment guidelines and patient adherence to the treatment plan.

CONCLUSIONS: Efforts by managed care pharmacists to improve the quality of care for patients with ACS and VTE can improve patient outcomes and reduce health care utilization and costs.

J Manag Care Pharm. 2008;14(6)(suppl S-a):S24-S27

Copyright@ 2008, Academy of Managed Care Pharmacy. All rights reserved.

\section{Author}

RANDALL D. SEIFERT, PharmD, is Senior Associate Dean at the University of Minnesota, Duluth.

AUTHOR CORRESPONDENCE: Randall D. Seifert, PharmD, Senior Associate Dean, University of Minnesota, Duluth, 232 Life Sciences, 1110 Kirby Dr., Duluth, MN 55812. Tel.: 218.726.6032;

Fax: 218.726.6500; E-mail: rseifert@umn.edu
$\Lambda$ cute coronary syndrome (ACS) and venous thromboembolism (VTE) place a large economic burden on society. The invasive cardiovascular procedures or intensive medical management that is required for patients with ACS and VTE can be costly due to the recurrence of deep vein thrombosis (DVT). Treatment and management of these conditions via practice guidelines can be challenging and controversial. Balancing cost with appropriate therapy is sometimes difficult to achieve.

\section{Acute Coronary Syndrome}

\section{Cost Implications}

ACS is a manifestation of coronary heart disease (CHD). In 2008, the estimated cost of CHD in the United States is \$156.4 billion, including $\$ 87.6$ billion for direct costs and $\$ 68.8$ billion for indirect costs. ${ }^{1}$

The scope of the problem for managed care organizations is significant as revealed in a retrospective analysis of claims for 13,731 managed care patients with a new onset of ACS. This well-designed study revealed that the average total cost during the first year after diagnosis was $\$ 22,529$ (mean: 9.75 months of follow-up), with $71 \%$ of these costs attributable to hospitalizations. ${ }^{2}$ More than one rehospitalization after the index event was required for 3,641 (26.5\%) of the 13,731 patients, thereby contributing significantly to the overall cost. ${ }^{2}$

In another retrospective study of claims for 16,321 managed care patients hospitalized for ACS, $21 \%$ of patients were rehospitalized for ischemic heart disease during a 1-year follow-up period at an average cost of $\$ 28,637 .^{3}$ Nearly one-half of the patients (46\%) underwent a revascularization procedure during the initial hospitalization. In the year after the index hospitalization, $90 \%$ of patients received lipid-lowering, antihypertensive, or antiarrhythmic medications, and $50 \%$ of patients received antiplatelet or anticoagulant medications.

Similarly, in a database of 3,258 managed care patients with ACS who underwent percutaneous coronary intervention, the rate of rehospitalization for restenosis was $12.5 \%{ }^{4}$ The mean cost over a 1-year period for rehospitalized patients was $\$ 31,954$, and the incremental cost for each patient with repeat revascularization was $\$ 24,955$.

\section{Clinical Outcomes}

Long-term use of dual antiplatelet therapy with aspirin and the thienopyridine clopidogrel is recommended for 12 months in patients with ACS, particularly in patients with drug-eluting coronary stents (see the article by Sobieszczyk in this supplement).,5 The causes and impact of patient nonadherence to prescribed antiplatelet therapy were evaluated in a prospective observational study of 500 patients with ACS and drug-eluting stents who received a prescription for thienopyridine therapy 
at the time of hospital discharge. ${ }^{7}$ Thirty days after discharge, $68(13.6 \%)$ patients had stopped taking the thienopyridine. The all-cause mortality rate within 1 year after discharge was significantly higher in patients who stopped thienopyridine therapy prematurely $(7.5 \%)$ than in those who continued therapy beyond 30 days $(0.7 \%, P<0.001)$. The number of patients needed to treat beyond 30 days with a thienopyridine to prevent 1 death is 15 patients. The rate of rehospitalizaton was also higher in patients who stopped thienopyridine therapy early (23\%) than it was in those who continued therapy $(14 \%, P=0.08)$. This result was not statistically significant and was likely related to the study methods; however, the absolute risk reduction of 9\% (number needed to treat $=11$ ) does shed light on some possible issues related to poor patient adherence and resultant clinical outcomes. These findings appear to underscore the preventable nature of rehospitalization and death and the importance of long-term antiplatelet therapy in patients with ACS and drug-eluting stents.

Compared with patients who continued thienopyridine therapy for at least 30 days, patients who discontinued therapy earlier were significantly older (64 years vs. 60 years, $P=0.03$ ), less likely to have a high school education ( $73 \%$ vs. $89 \%, P<0.001)$ and be married ( $56 \%$ vs. $71 \%, P=0.01$ ), and more likely to have preexisting cardiovascular illnesses (49\% vs. $30 \%, P=0.003)$ and avoid health care due to cost ( $24 \%$ vs. $13 \%, P=0.02) .{ }^{7}$ Patients who discontinued therapy prematurely were also less likely to have received discharge instructions ( $88 \%$ vs. $95 \%, P=0.05)$ or a referral for cardiac rehabilitation $(50 \%$ vs. $64 \%, P=0.03)$ than patients who continued therapy. These findings have important implications regarding the identification of patients with ACS so as to counsel them about the importance of adhering to prescribed antiplatelet therapy on a long-term basis to prevent death and costly hospitalizations.

\section{Venous Thromboembolism}

\section{Cost Implications}

Venous thromboembolism-DVT and pulmonary embolism (PE) - is costly because of the high rate of DVT recurrence (8\%-17\% within 2 years) and the costs associated with longterm sequelae, particularly post-thrombotic syndrome (see the article by Fanikos in this supplement). ${ }^{8,9}$ In a retrospective pharmacoeconomic analysis, extrapolation of data from patient and administrative records for managed care patients with acute proximal DVT suggests that the annual costs of treatment in 1997 amounted to $\$ 1.5$ billion in the United States. ${ }^{10}$

A more comprehensive, controlled, retrospective observational study was conducted of administrative claims data for 26,958 managed care patients with DVT, PE, or both and patients with possible post-thrombotic syndrome. ${ }^{11}$ The mean annualized total health care costs for DVT, PE, or both were $\$ 33,200, \$ 31,300$, and $\$ 38,300$, respectively, compared with $\$ 2,800$ for matched control patients. The mean annualized total health care costs were $\$ 47,600$ in patients with post-thrombotic syndrome and
$\$ 35,900$ in patients without the syndrome (i.e., the incremental cost of post-thrombotic syndrome was $\$ 11,700$ ).

\section{Clinical Outcomes}

Several issues related to the optimal use of anticoagulant agents for the treatment of VTE have been raised. Quality improvement efforts by managed care pharmacists should address these issues.

The feasibility, safety, and efficacy of subcutaneous low-molecular-weight heparin (LMWH) therapy in outpatients instead of standard intravenous unfractionated heparin (UFH) in inpatients has been demonstrated in randomized, controlled, and prospective cohort studies of patients with DVT, PE, or both, although fewer data are available on the use of LMWH in the treatment of PE than of DVT. ${ }^{12-15}$ Pregnancy, a history of VTE, a high likelihood of patient noncompliance, and the presence of thrombophilic or serious comorbid conditions were among exclusion criteria in these studies. Nevertheless, most patients with clinically stable VTE are candidates for outpatient LMWH therapy.

Low rates of DVT recurrence (3\%-7\%) and bleeding (3\%-4\%) were observed in cost-effectiveness studies of LMWH for the treatment of DVT. ${ }^{16,17}$ The acquisition cost is higher for LMWH than for UFH. Cost savings, primarily from a shortened length of hospital stay, were associated with the use of LMWH instead of UFH in a study of 300 patients with DVT. ${ }^{16}$ In another costeffectiveness analysis, the cost of initial care was higher in patients treated with LMWH than in patients treated with UFH, but this higher initial cost was partly offset by lower costs for early complications. ${ }^{17}$

In a prospective study of an outpatient DVT treatment protocol at a staff model health maintenance organization, 102 (61\%) of 167 patients with VTE were eligible for and received LMWH therapy as outpatients. ${ }^{18}$ Their VTE recurrence rate was 1.9\% versus $4.1 \%$ in a cohort of patients treated previously with UFH as inpatients. None of the outpatients or inpatients experienced major bleeding. The average length of hospital stay was 0.79 days for outpatients compared with 5.3 days for inpatients. Total costs for outpatients $(\$ 229,270)$ were roughly one half as much as for inpatients $(\$ 484,288)$. The overall outpatient care experience was rated very good or excellent by 41 (84\%) of 49 outpatients due to avoidance of hospitalization, less disruption in routine, and a quick return to work. The potential for cost savings and patient satisfaction without compromising efficacy or safety associated with the use of LMWH on an outpatient basis for treatment of patients with VTE should be considered in designing managed care benefits. Additional randomized controlled trials are needed to support the potential benefits of LMWH in outpatients for the treatment of clinically stable PE.

\section{Managed Care Plan Benefit Design}

Managed care plan benefits should include provisions for the optimal duration of therapy with anticoagulants for the 
prevention and treatment of VTE. Three months of treatment with a vitamin $\mathrm{K}$ antagonist (e.g., warfarin) are recommended by the American College of Chest Physicians for patients with a first episode of VTE due to a transient, reversible cause because a shorter duration of therapy (4-6 weeks) is associated with an increased risk for VTE recurrence. ${ }^{19-22}$ At least 6-12 months of anticoagulant treatment are recommended for an idiopathic first DVT episode, and treatment for an indefinite period is recommended for patients with 2 or more VTE episodes and for patients with a first episode of VTE and cancer or certain inherited causes of thrombophilia (see the article by Fanikos in this supplement). ${ }^{19}$

In patients with acute DVT, treatment with LMWH for 3-6 months is at least as effective in preventing recurrent DVT as a vitamin $\mathrm{K}$ antagonist with doses adjusted to maintain an international normalized ratio (INR) of 2.0-3.0, and LMWH therapy is associated with a lower risk for minor bleeding. ${ }^{23-25} \mathrm{In}$ a randomized, open-label study of 672 patients with cancer and acute DVT, 6 months of treatment with the LMWH dalteparin 200 units per kg subcutaneously once daily for 1 month followed by 150 units per kg subcutaneously once daily for 5 months was compared with dalteparin 200 units per kg subcutaneously once daily for 5-7 days followed by a vitamin $\mathrm{K}$ antagonist with the dose adjusted to maintain an INR of 2.5 for 6 months. ${ }^{25}$ The probability of DVT recurrence after 6 months was $9 \%$ in the dalteparin-only group and $17 \%$ in the dalteparin-vitamin $\mathrm{K}$ antagonist group, providing a difference that is significant $(P=0.002)$. The incidence of major bleeding was not significantly different at $6 \%$ and $4 \%$, respectively $(P=0.27)$. The number of patients needed to treat with dalteparin only for 6 months to prevent 1 case of DVT recurrence is 13. Thus, long-term LMWH treatment for acute DVT is safe and effective and is preferred over warfarin for patients with cancer.

\section{Opportunities for Managed Care Pharmacists}

Evidence-based guidelines provide a framework for managed care interventions and quality improvement efforts. Recently updated guidelines for the management of ACS are available from the American College of Cardiology and American Heart Association. ${ }^{5,6}$ Managed care pharmacists can familiarize themselves with guidelines for ACS and VTE as well as other recent research findings related to the management of these conditions by using Web-based educational programs available from ASHP Advantage (www.ashpadvantage.com/clot/).

Incorporating new guidelines and ensuring adherence to the guidelines in managed care can be a challenge without making major changes to the chronic care delivery model. Advances in information technology can facilitate the incorporation of and promote adherence to new treatment guidelines. Research involving evaluation of managed care operations is needed to identify strategies to improve clinician knowledge of and adherence to treatment guidelines and patient adherence to prescribed therapy. Patient-centered management approaches, disease management programs that promote medication adherence, and pharmacist-based medication therapy management with reconciliation between the inpatient and outpatient settings are possible strategies to achieve these goals.

One such strategy was outlined in the Asheville Project, which is a patient-centered management model for improving medication adherence in patients with chronic conditions (e.g., diabetes, asthma, hypertension, dyslipidemia) that, in theory, could be applied to patients with ACS or VTE. ${ }^{26}$ Improved patient outcomes and substantial reductions in health care utilization, sick days, and costs have been realized using this model. The pharmacist serves as a health coach by directing the patient to health information and monitoring drug therapy.

In addition, Health Effectiveness Data Information Set (HEDIS) criteria are used by most managed care plans to measure health care quality. Only 1 HEDIS criterion-beta-blocker use after acute myocardial infarction-relates directly to patients with ACS, and none pertain to VTE prevention or treatment. ${ }^{27}$ Other HEDIS criteria address the management of diabetes mellitus complicated by high cholesterol and high blood pressure, which are CHD risk factors.

Implementing new quality measures with pay-for-performance financial incentives is another approach that managed care pharmacists can use to improve the quality of care for patients with ACS or VTE. A reduction in health care costs could be realized. Modifying the managed care plan benefit structure to reduce premiums or copayments for medications is a possible approach to promote program participation and adherence to prescribed therapy by patients.

This review found several good-quality studies that are relevant to managed care pharmacy practice; however, there is a need to initiate studies that document best practice interventions, whether they are clinical or related to benefit design. The need to identify and implement strategies to improve clinician adherence to treatment guidelines and patient adherence to prescribed therapy presents managed care pharmacists with both a challenge and an opportunity. Improvements in the quality of care and clinical outcomes in patients with ACS or VTE can be achieved through managed care pharmacist efforts.

\section{Conclusions}

Some of the preventable morbidity, mortality, and costs associated with ACS and VTE can be avoided by improving the use of anticoagulant and antiplatelet therapy in patients with these conditions. Efforts by managed care pharmacists can contribute to these goals.

\section{DISCLOSURES}

Randall D. Seifert discloses that there was no financial relationship or financial interest relating to the topic of this activity. Seifert was responsible for the entire study concept and design of this article. He performed all of the data collection, data interpretation, writing, and revision of this article. 


\section{REFERENCES}

1. American Heart Association/American Stroke Association. Heart disease and stroke statistics—2008 update: a report from the American Heart Association Statistics Committee and Stroke Statistics Subcommittee. Available at: www.americanheart.org/downloadable/ heart/1200082005246HS_Stats\%202008.final.pdf. Accessed March 14, 2008 .

2. Etemad LR, McCollam PL. Total first-year costs of acute coronary syndrome in a managed care setting. J Manag Care Pharm. 2005;11:300-06. Available at: www.amcp.org/data/jmcp/research_300_306.pdf.

3. Menzin J, Wygant $G$, Hauch $O$, et al. One-year costs of ischemic heart disease among patients with acute coronary syndromes: findings from a multi-employer claims database. Curr Med Res Opin. 2008;24:461-68.

4. Clark MA, Bakhai A, Pelletier EM, et al. Clinical and economic effects of coronary restenosis after percutaneous coronary intervention in a managed care population. Manag Care. 2005;14:42-44, 46-51.

5. Antman EM, Hand M, Armstrong PW, et al. 2007 Focused update of the ACC/AHA 2004 guidelines for the management of patients with ST-elevation myocardial infarction: a report of the American College of Cardiology/ American Heart Association Task Force on Practice Guidelines. Circulation. 2008;117:296-329

6. Anderson JL, Adams CD, Antman EM, et al. ACC/AHA 2007 guidelines for the management of patients with unstable angina/non ST-elevation myocardial infarction: a report of the American College of Cardiology/ American Heart Association Task Force on Practice Guidelines. Circulation. 2007;116:e148-304

7. Spertus JA, Kettelkamp R, Vance C, et al. Prevalence, predictors, and outcomes of premature discontinuation of thienopyridine therapy after drug-eluting stent placement: results from the PREMIER registry. Circulation. 2006;113:2803-09.

8. Cushman M, Tsai AW, White RH, et al. Deep vein thrombosis and pulmonary embolism in two cohorts: the longitudinal investigation of thromboembolism etiology. Am J Med. 2004;117:19-25.

9. Prandoni P, Villalta S, Bagatella P, et al. The clinical course of deep-vein thrombosis. Prospective long-term follow-up of 528 symptomatic patients. Haematologica. 1997;82:423-28

10. Spyropoulos AC, Hurley JS, Ciesla GN, et al. Management of acute proximal deep vein thrombosis: pharmacoeconomic evaluation of outpatient treatment with enoxaparin vs inpatient treatment with unfractionated heparin. Chest. 2002;122:108-14.

11. MacDougall DA, Feliu AL, Boccuzzi SJ, et al. Economic burden of deepvein thrombosis, pulmonary embolism, and post-thrombotic syndrome. Am J Health Syst Pharm. 2006;63(20 suppl 6):S5-15.

12. Levine M, Gent M, Hirsh J, et al. A comparison of low-molecular-weight heparin administered primarily at home with unfractionated heparin administered in the hospital for proximal deep-vein thrombosis. $N$ Engl J Med. 1996;334:677-81.

13. Koopman MM, Prandoni P, Piovella F, et al. Treatment of venous thrombosis with intravenous unfractionated heparin administered in the hospital as compared with subcutaneous low-molecular-weight heparin administered at home. The Tasman Study Group. N Engl J Med. 1996;334:682-87.
14. Kovacs MJ, Anderson D, Morrow B, et al. Outpatient treatment of pulmonary embolism with dalteparin. Thromb Haemost. 2000;83:209-11.

15. Hull RD, Raskob GE, Brant RF, et al. Low-molecular-weight heparin vs heparin in the treatment of patients with pulmonary embolism. AmericanCanadian Thrombosis Study Group. Arch Intern Med. 2000;160:229-36.

16. O'Brien B, Levine M, Willan A, et al. Economic evaluation of outpatient treatment with low-molecular-weight heparin for proximal vein thrombosis. Arch Intern Med. 1999;159:2298-304.

17. Gould MK, Dembitzer AD, Sanders GD, et al. Low-molecular-weight heparins compared with unfractionated heparin for treatment of acute deep venous thrombosis. A cost-effectiveness analysis. Ann Intern Med. 1999; 130:789-99.

18. Spyropoulos AC. Outpatient-based treatment protocols in the management of venous thromboembolic disease. Am J Manag Care. 2000; 6(20 suppl):S1034-44.

19. Büller HR, Agnelli G, Hull RD, et al. Antithrombotic therapy for venous thromboembolic disease: the Seventh ACCP Conference on Antithrombotic and Thrombolytic Therapy. Chest. 2004;126(3 suppl):401S-28S.

20. Research Committee of the British Thoracic Society. Optimum duration of anticoagulation for deep-vein thrombosis and pulmonary embolism. Lancet. 1992;340:873-76

21. Schulman S, Rhedin AS, Lindmarker P, et al. A comparison of six weeks with six months of oral anticoagulant therapy after a first episode of venous thromboembolism. Duration of Anticoagulation Trial Study Group. N Engl J Med. 1995;332:1661-65.

22. Levine MN, Hirsh J, Gent M, et al. Optimal duration of oral anticoagulant therapy: a randomized trial comparing four weeks with three months of warfarin in patients with proximal deep vein thrombosis. Thromb Haemost. 1995;74:606-11.

23. Hull R, Pineo GF, Mah A, et al. A randomized trial evaluating long-term low-molecular-weight heparin therapy for three months versus intravenous heparin followed by warfarin sodium [abstract]. Blood. 2002;100:148a.

24. Hull R, Pineo GF, Mah A, et al. Safety and efficacy results for a study investigating the long-term out-of-hospital treatment of patients with proximal-vein thrombosis using subcutaneous low-molecular-weight heparin versus warfarin [abstract]. Thromb Haemost. 2001;(suppl):abstract OCl647.

25. Lee AY, Levine MN, Baker RI, et al. Low-molecular-weight heparin versus a coumarin for the prevention of recurrent venous thromboembolism in patients with cancer. N Engl J Med. 2003;349:146-53.

26. Heinze C. The Asheville Project now: employers nationwide adopt costsaving health care model. Available at: www.pharmacytimes.com/issues/ articles/SUPP_2007-10_7594.asp. Accessed March 15, 2008.

27. National Committee for Quality Assurance. HEDIS 2008 summary table of measures, product lines and changes. Available at: www.ncqa.org/ Portals/0/HEDISQM/HEDIS2008/2008_Measures.pdf. Accessed March 15, 2008. 